\title{
Gravitational wave bursts induced by r-mode spin-down of hybrid stars
}

\author{
A. Drago ${ }^{1}$, G. Pagliara ${ }^{1}$, and Z. Berezhiani ${ }^{2}$ \\ 1 Dipartimento di Fisica, Università di Ferrara and INFN, Sezione di Ferrara, 44100 Ferrara, Italy \\ e-mail: drago@fe.infn.it \\ 2 Dipartimento di Fisica, Università di L'Aquila and INFN, Laboratori Nazionali del Gran Sasso, 67010 L'Aquila, Italy
}

Received 20 April 2005 / Accepted 23 August 2005

\begin{abstract}
We show that sudden variations in the composition and structure of a hybrid star can be triggered by its rapid spin-down, induced by r-mode instabilities. The discontinuity of this process is due to the surface tension between hadronic and quark matter and in particular to the overpressure needed to nucleate new structures of quark matter in the mixed phase. The consequent mini-collapses in the star can produce highly energetic gravitational wave bursts. The possible connection between the predictions of this model and the burst signal found by EXPLORER and NAUTILUS detectors during 2001 is also investigated.
\end{abstract}

Key words. gravitational waves - equation of state - stars: neutron - stars: evolution - stars: rotation - stars: interiors

\section{Introduction}

The analysis of the data collected by the Gravitational Wave antennas EXPLORER and NAUTILUS during 2001 shows coincidences between the signals detected by these two resonant bars (Astone et al. 2002; Coccia et al. 2004). In particular, an excess of coincidences with respect to the background is concentrated around sidereal hour four, which corresponds to the orientation for which the sensitivity of the bars is maximal for a signal coming from the direction of the galactic center. Although the statistical significance of this signal is debated (see Finn 2003; Astone et al. 2003b; Astone et al. 2003a), it is interesting to investigate the possible origin of the inferred signal using existing models of $\mathrm{GW}$ emission. In particular we will concentrate on the ones associated with instability modes of a rotating compact star. It is important to remark that in the analysis performed on the data from the two gravitational antennas only impulsive signals have been searched for, with a typical duration of $\sim 10 \mathrm{~ms}$. In the literature an extensive analysis has been performed concerning steady sources and periodic GW emission (Andersson et al. 2002; Wagoner 2002; Reisenegger \& Bonacic 2003). In this paper we will explore the possibility that a transient GW burst is triggered by the steady emission of GWs generated by r-mode instabilities. In particular, we will show that the almost continuous dragging of angular momentum from the star can induce a sudden variation in its structure and composition, generating a few bursts of GWs.

The first ingredient of our model are the so called r-mode instabilities, which are very efficient in dragging angular momentum from a rapidly rotating compact star (Andersson 1998; Friedman \& Morsink 1998). A rapid spindown deeply affects the structure and composition of the star. This problem has been discussed in detail in a few papers in the past years (see Glendenning et al. 1997; Chubarian et al. 2000), where it has been shown that, using rather standard values for the model parameters, the composition of an hybrid hadronic-quark star can be dramatically modified when the rotational frequency changes in a range centered around a few hundred Hertz. In particular, during the spin-down era the central density increases and a larger amount of matter is converted into a mixed hadron-quark phase (MP) or into pure quark matter. The new idea we discuss in this paper is the effect of a surface tension at the interface between hadronic and quark matter. If the surface tension is not vanishing, the formation of new structures in the MP can be delayed. A similar idea has been discussed recently in connection with the transition from a metastable hadronic star into a stable hybrid or quark star, showing that a huge amount of energy can be released in the process of conversion of bulk hadronic matter into quark matter (Berezhiani et al. 2003; Drago et al. 2004; Bombaci et al. 2004) ${ }^{1}$. The mechanism discussed in the present paper is also based on metastability, but it

\footnotetext{
1 A similar idea has recently been discussed in Harko et al. (2004). There, the possibility that the spin down can trigger a phase transition is analyzed taking into account the effect of the surface tension. In Harko et al. (2004) the angular momentum of the star is dragged by the magnetic dipole radiation which can be an efficient mechanism of spin down only in presence of a strong magnetic field.
} 
involves phase transitions in the MP, which are responsible for a relatively large modification of the stellar structure without a large energy release. We suggest the possibility that the formation of the MP can take place in several steps, in each of which the radius and the oblateness of the star change only by a relatively small amount. A crucial observation is that the conversion process from hadronic matter into MP will start in one point inside a spheroidal layer of metastable matter (to be discussed later) and will propagate inside that same layer at finite velocity. In this way the burning process generates a large non-radial oscillation of the star. During this period a strong GW emission takes place, and we will discuss its possible connection with the analysis of the gravitational bar data by the ROG Collaboration ${ }^{2}$.

\section{R-mode induced spin down}

In the literature, two scenarios have been discussed concerning a steady GW emission due to r-mode instabilities. The first scenario is based on the emission of GWs from a hot and rapidly spinning compact stellar object, which has not lost its angular momentum in the very first part of its existence after the supernova explosion. This is possible if the bulk viscosity is large for temperatures of the order of $\sim 10^{9} \mathrm{~K}$ or higher, so that $\mathrm{r}$-modes are damped until the temperature drops below $\sim 10^{9} \mathrm{~K}$. Possible candidates are quark stars (Andersson et al. 2002), hyperonic stars (Lindblom \& Owen 2002) or hybrid stars (Drago et al. 2005). The second scenario involves older stars that are reaccelerating due to mass accretion from a companion. In this way a sort of "cycle" can develop (see Fig. 1), in which the star periodically goes through the following steps: 1) mass accretion with increase of angular velocity; 2) instability due to r-mode excitations with reheating due to bulk viscosity; 3) loss of angular momentum with emission of GWs. The reheating due to bulk viscosity during phase 2 ) is so efficient that, if the instability region is reached from the low-temperature side, the star rapidly reheats and reaches the high-temperature side of the instability region (Reisenegger \& Bonacic 2003) ${ }^{3}$.

Let us introduce the equations regulating the process of $\mathrm{GW}$ emission in the two scenarios discussed above. Here we follow the formalism of Wagoner (2002) and Kinney \& Mendell (2003), where the scheme proposed by Owen et al. (1998) has been refined ${ }^{4}$. The evolution equations read:

$\frac{\dot{\alpha}}{\alpha}=\frac{-1}{t_{v}}-\left(1+\frac{3 \alpha^{2} \tilde{J}}{2 \tilde{I}}\right)\left(\frac{1}{t_{g}}\right)-\frac{\dot{M}}{2 \tilde{I} \Omega}\left(\frac{G}{M R^{3}}\right)^{\frac{1}{2}}$

\footnotetext{
2 Another possible relation between phase transitions and GW emissions is discussed in Miniutti et al. (2003). In that paper phase transitions, associated with density discontinuities, can excite the socalled g-modes and large non-radial oscillations can develop.

3 Also neutron stars can recycle, as suggested in Levin (1999), Andersson et al. (2000), Heyl (2002).

${ }^{4}$ We assume that the r-mode canonical angular momentum contributes zero physical angular momentum to the star. This is a sensible approximation as results both from explicit calculations (Levin \& Ushomirsky 2001) and also from the observation that the r-modes saturate quickly and after saturation no physical angular momentum can go into them (Wagoner 2002; Kinney \& Mendell 2003).
}
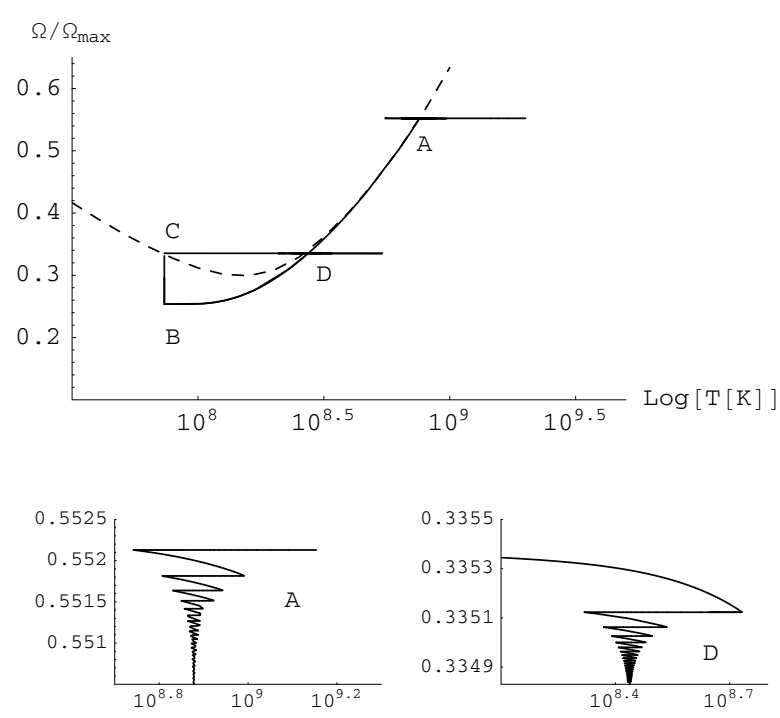

Fig. 1. r-mode instability window (upper panel). Coming from high temperatures (A) the star loses its angular momentum until it exits the instability region (B) (first scenario). By mass accretion the star can be re-accelerated until it reaches the instability window from the low temperature side $(\mathrm{C})$. The excitation of r-modes instability produces a fast reheating of the star due to bulk viscosity dissipation (D) (second scenario). In the lower panel a magnification of the regions indicated by (A) and (D) is displayed. Here we used a mass accretion rate of $10^{-8} M_{\odot}$ per year.

$\dot{\Omega}=\frac{\dot{M}}{\tilde{I}}\left(\frac{G}{M R^{3}}\right)^{1 / 2}-\frac{\dot{M} \Omega}{M}+3 \Omega \alpha^{2} \frac{\tilde{J}}{\tilde{I}}\left(\frac{1}{t_{g}}\right)$
$\dot{E}_{\text {thermal }}=\dot{E}_{\text {accretion }}+\dot{E}_{\text {viscosity }}-\dot{E}_{\text {neutrino. }}$.

Here $\alpha$ is the dimensionless amplitude of the r-mode, $1 / t_{\mathrm{v}} \equiv$ $1 / t_{\mathrm{s}}+1 / t_{\mathrm{b}}, t_{\mathrm{g}}, t_{\mathrm{s}}$ and $t_{\mathrm{b}}$ are time scales associated with GW emission, to shear and to bulk viscosity damping, respectively. $\tilde{I}$ and $\tilde{J}$ are dimensionless values of the moment of inertia and of the angular momentum (for all details see e.g. Andersson et al. 2002). Equation (1) describes the damping of r-modes due to viscosity, Eq. (2) describes the variation of the angular momentum of the star in presence of the torque due to gravitational wave emission and to mass accretion and, finally, Eq. (3) describes the thermal evolution given by the contributions of the reheating due to mass accretion, shear and bulk viscous dissipation of the r-modes and of the cooling due to neutrino emission. Obviously, in the first scenario depicted above, mass accretion is not present. To compute the time scale associated with bulk viscosity for hybrid stars we use the results of Drago et al. (2005) in which the viscosity of MP has been computed. They showed that the viscosity of MP is of the same order of magnitude as the viscosity of pure quark matter if superconducting gaps are not present or it is reduced by a factor of $\sim 10$ if a color superconducting $2 \mathrm{SC}$ gap is taken into account (see Madsen 2000). Concerning the value of shear viscosity, we have taken into account not only the contribution associated with pure quark matter (Andersson et al. 2002), but also the contribution associated with the viscous boundary layer which is present in a star having a crust made of nucleonic matter (Bildsten \& Ushomirsky 1999). In Fig. 2 we plot the dimensionless amplitude $h$ and the frequency $f$ of the GWs 

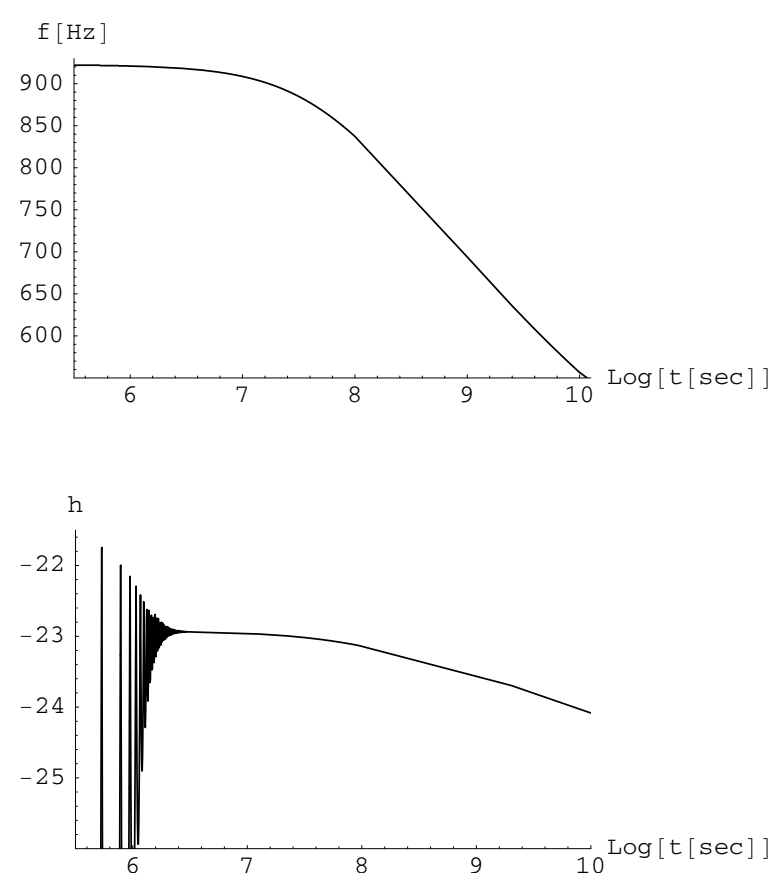

Fig. 2. The frequency (upper panel) and amplitude (lower panel) of the GW signal emitted by r-mode instabilities as functions of time. Here the compact star is approaching the instability window from the high temperature side (first scenario). The computed amplitude corresponds to a distance of $1 \mathrm{kpc}$.

emitted by a star in the first scenario. In Fig. 3, the same quantities are displayed for the second scenario. In this figure, a mass accretion rate of order $10^{-8} M_{\odot}$ per year has been assumed. If the value of the accretion rate is reduced, the star re-enters the instability window at smaller temperatures and larger values of the angular velocity. In this way, the GW emission in the second scenario would be similar to the one obtained in the first scenario. Finally, let us remark that the same compact star can enter first the instability window associated with the first scenario, when the star is relatively young, and, after some time, the star becomes again unstable due to mass accretion as described in the second scenario. These possibilities are displayed in Fig. 1.

As it can be seen in Figs. 2 and 3, in both scenarios the initial part of the signal decomposes into bursts lasting a few minutes and separated by periods of a few days (or months) of quiescence. During this initial phase of the emission, the star follows a trajectory in the temperature - angular velocity plane, oscillating around the instability line displayed in Fig. 1 (see also Andersson et al. 2002). After this phase, which can last months or years, the angular momentum is dragged almost continuously and the signal becomes steady for hundreds of years until the star finally exits the instability region. In principle, the first part of the signal having an amplitude $h \sim 10^{-22}$ for a source located at $1 \mathrm{kpc}$ could be detected by resonant bars. The main difficulty associated with the search for this type of periodic signal is due to the almost monocromaticity of the GW emission which could be detected only by a dedicated search. For instance, in the case of the pulsar J1939+2134, a dedicated search has been performed for a signal having a frequency
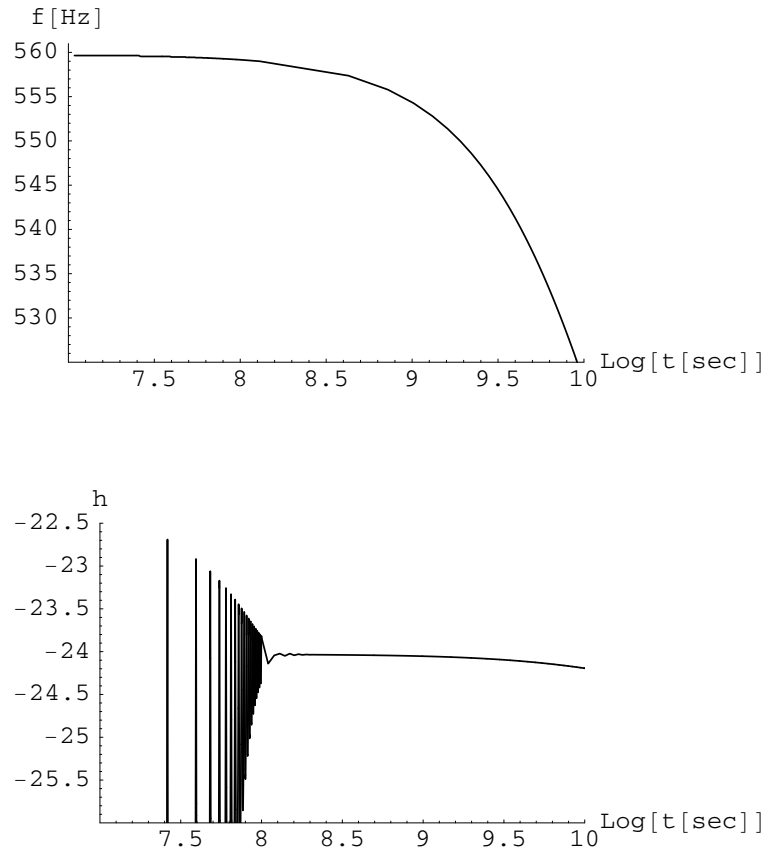

Fig. 3. Same as in Fig. 1. Here the compact star is approaching the instability window from the low temperature side after the "recycling" process (second scenario).

twice the rotation frequency of the star (which would correspond to the signal emitted by a stellar object having a nonaxisimmetric shape), with a null result (see Abbott et al. 2004). On the other hand, the signal associated with r-modes would mainly be emitted at a frequency $f_{\mathrm{g}}=2 \Omega / 3 \pi$.

\section{Toy-model for a rotating star}

The r-mode mechanism we have discussed so far produces a steady emission of periodic GWs and therefore it does not correspond to the signal discussed in Astone et al. (2002), Coccia et al. (2004). The new idea we are now introducing is a possible connection between the rapid dragging of the angular momentum and discontinuous changes of the structure of the star. It is worth remarking that $r$-modes are a very efficient way of reducing the angular momentum of a compact star. Although it will not be discussed here, also a strong magnetic field as such the one present in magnetars can efficiently drag the angular momentum of the star by dipole magnetic radiation (Harko et al. 2004). As shown in Glendenning et al. (1997), Chubarian et al. (2000), when a hybrid star slows down, its density increases until the first critical density is reached at the center of the star and MP starts being produced. A further reduction of the angular momentum allows the star to increase the fraction of MP occupying its core, until the central density reaches the second critical value. At that point pure quark matter starts being produced. During all the spin-down process the radius of the star decreases by several kilometers. This gradual modification of the structure of the star can take place discontinuously in several (small) steps if the effect of a non-vanishing surface tension is taken into account. The inner regions of the star in which MP will later form becomes firstly metastable. The formation of germs of stable MP proceeds through quantum tunneling. 
The probability of this transition strongly depends on the value of overpressure. As shown in Iida \& Sato (1998), Berezhiani et al. (2003), Drago et al. (2004), Bombaci et al. (2004) the nucleation time needed to form new structures of quark matter can be very long if the overpressure is not large. When the overpressure reaches the critical value, in one randomly chosen site inside the metastable layer a new drop of quark matter forms. As it will be shown, the process of conversion of hadronic into quark matter propagates with finite velocity $v_{\mathrm{c}}$ inside the star and a sudden modification of the composition and of the structure of the star occurs during a timescale $\sim R / v_{\mathrm{c}}$. During this period non-radial modes develop and a few bursts of GWs can be emitted until a new equilibrium configuration is reached.

To give a qualitative estimate of the magnitude of the oscillations and therefore of the amplitude of the GWs emitted we use the toy model proposed in Heiselberg \& Hjorth-Jensen (1998). We model the hybrid star as a spheroid containing a MP core with uniform density $\rho_{2}$ and a crust of nuclear matter with uniform density $\rho_{1}$. The pressure at which the phase transition from the first to the second component takes place is a parameter $P_{0}$. The Newtonian hydrostatic equation reads:

$\frac{1}{\rho} \frac{\mathrm{d} P}{\mathrm{~d} a}=-G \frac{m(a)}{a^{2}}+\frac{2}{3} \Omega^{2} a$,

where

$m(a)=4 \pi \int_{0}^{a} \rho\left(a^{\prime}\right) a^{\prime 2} \mathrm{~d} a^{\prime}$.

Here the "effective radius" $a$ appears, which is related to the position inside the star by the equation:

$r=a\left(1-\epsilon P_{2}(\cos \theta)\right)$,

where $\epsilon=\frac{5}{4} \frac{\Omega^{2}}{2 \pi G \rho_{1}}$ and $P_{2}(\cos \theta)$ is the 2nd Legendre polynomial. Equation (4) is then solved and in this way the pressure inside the star is analytically determined for a given angular velocity. In Fig. 4 the structure of a rotating star is displayed. Comparing with results based on the actual solution of the equilibrium equation for a rotating compact object (see e.g. Glendenning et al. 1997; Chubarian et al. 2000), we see that the toy model is at least qualitatively in agreement with the exact solution. Unfortunately, the toy model badly underestimates the quantitative effect of the spin-down on the radius and composition of the star. For instance, in the toy model we get reductions of the radius of the order of $0.5 \mathrm{~km}$, while the calculations based on realistic equations of state give reductions typically of the order of a few km (Glendenning et al. 1997; Chubarian et al. 2000). The same problem arises for the estimate of the fraction of the volume of the star occupied by MP: the toy model gives an increase of this quantity of roughly $20 \%$, while more realistic calculations give a significantly larger value. It is clear that we are at the moment only aiming at a qualitative study of the proposed mechanism and that refined calculations are needed in order to obtain a more precise estimate.

The question we want to numerically investigate within the toy model is the following: given a specific equation of state (in our case fixing the values of $\rho_{1}, \rho_{2}$ and $P_{0}$ ) and a value for the surface tension $\sigma$, what variation of the angular velocity $\Delta \Omega / \Omega$ is large enough to trigger the formation of a critical drop of

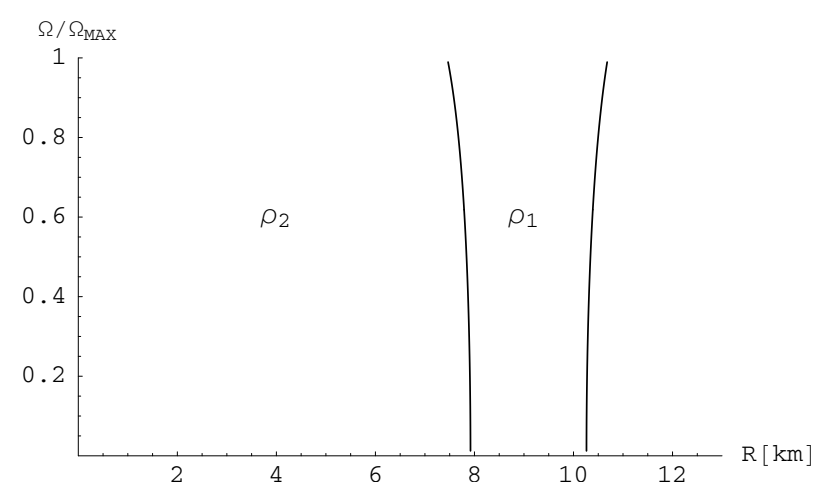

Fig. 4. The total radius of the star and the radius of the inner core of MP shown as functions of the angular velocity in the toy model.

quark matter, in a time scale of order days or years? The crucial ingredient in this calculation is the relation between the overpressure $\Delta P / P$ and $\Delta \Omega / \Omega$. The overpressure is determined computing, for the same element of fluid, the difference between the value of the pressure after and before the slow down (Lagrangian perturbation). In particular, we are interested in the value of the overpressure in the region immediately surrounding the core of already formed MP. In Fig. 5 we show the overpressure, in the above defined region, for a value $\Delta \Omega / \Omega=0.05$ and for various values of $\Omega$. As it appears, $\Delta P / P \sim \alpha \Delta \Omega / \Omega$, with $\alpha \sim 0.3 \div 1.4$, and the larger values correspond to the faster rotating stars. It is also interesting to notice that, as the star slows down the layer in which new MP is formed moves to outer regions and the thickness of the layer shrinks. In Fig. 6 the overpressure inside the star is displayed for a fixed value of $\Omega$ and $\Delta \Omega / \Omega$. Notice that the value of the overpressure is larger in the layer around the MP core and therefore in that region the conversion will take place with a higher probability.

We need now to compute the nucleation time for the obtained value of the overpressure and a given value of $\sigma$. This can be done following the formalism developed in Iida \& Sato (1998), Berezhiani et al. (2003) and based on quantum tunneling. Within the toy model, a nucleation time of order days can be obtained, for values of the overpressure as the one displayed in Fig. 5 and using values of $\sigma \sim$ a few $\mathrm{MeV} / \mathrm{fm}^{2}$. These values of $\sigma$ are of the same order of the one estimated in the MIT bag model (Berger \& Jaffe 1987). They are also not far from the values investigated in Berezhiani et al. (2003), Drago et al. (2004), Bombaci et al. (2004). We would like to stress again that refined calculations are needed in order to derive a quantitative estimate of the nucleation time. The most artificial feature of the toy model is probably related to the absence of modifications of the already-formed MP during the spin-down process. In a realistic model, not only the volume occupied by MP increases during the spin-down, but also new structures form, with an evolution of the existing structures from drops, to rods, to slabs as the star slows down (Glendenning 1997). 


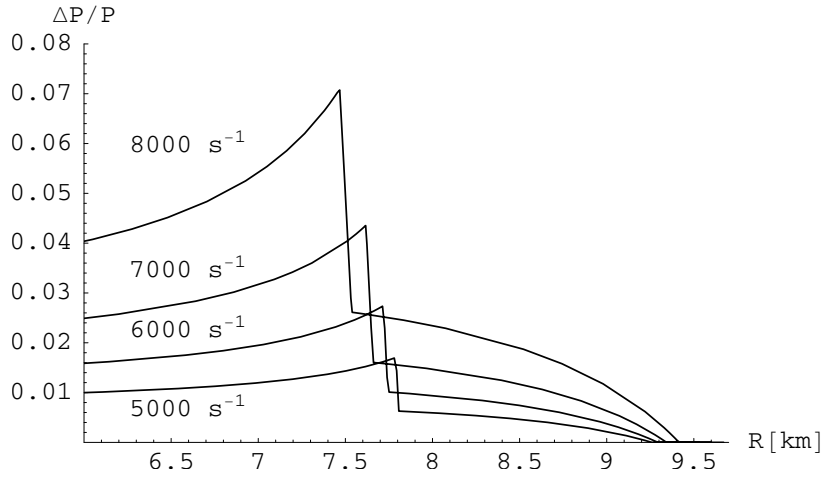

Fig. 5. The value of the overpressure as a function of the effective radius, for four values of the angular velocity. Here $\Delta \Omega / \Omega=0.05$.

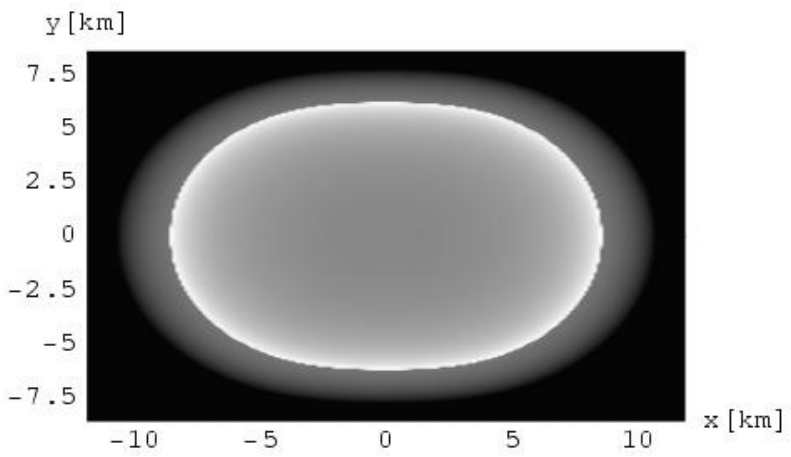

Fig. 6. The value of the overpressure inside the star, for $\Omega=5000 \mathrm{~s}^{-1}$ and $\Delta \Omega / \Omega=0.05$. Lighter areas correspond to higher values of the overpressure.

\section{Emission of GW bursts}

\subsection{Time structure of the bursts}

We can now describe more precisely the GW emission in the mechanism here introduced. As it has been shown in Figs. 2-3, it is possible to reduce the angular velocity of the star by some $10-20 \%$ in $\sim 10$ years via emission of periodic GWs induced by r-mode instabilities. This reduction corresponds to an increase of the inner pressure by roughly the same percent. As we have seen, when an overpressure of the order of a few percent is reached, the star will readjusts forming a new region of MP. Therefore, we can expect a few periods of GW burst activity in 10 years. In this model the mechanism of emission of GWs is similar to the one invoked in connection with the so-called soft-gamma repeaters (see de Freitas Pacheco 1998). In particular, in both models the burst of GWs is due to a starquake activity. The main difference between the two models is that in the soft-gamma repeaters case, the origin of the sudden change of the structure of the star is related to the maximum shear stress that the crust can bear before cracking. In the model proposed in the present paper, the role played by the rigidity of the crust is now played by the surface tension at the interface between hadronic and quark matter. As we will show, it is possible in our model to obtain more violent oscillations of the star and therefore more energetic GW bursts. This is not surprising since the physics underlying the soft-gamma repeaters model is based on a metastability related to the typical energy scale of

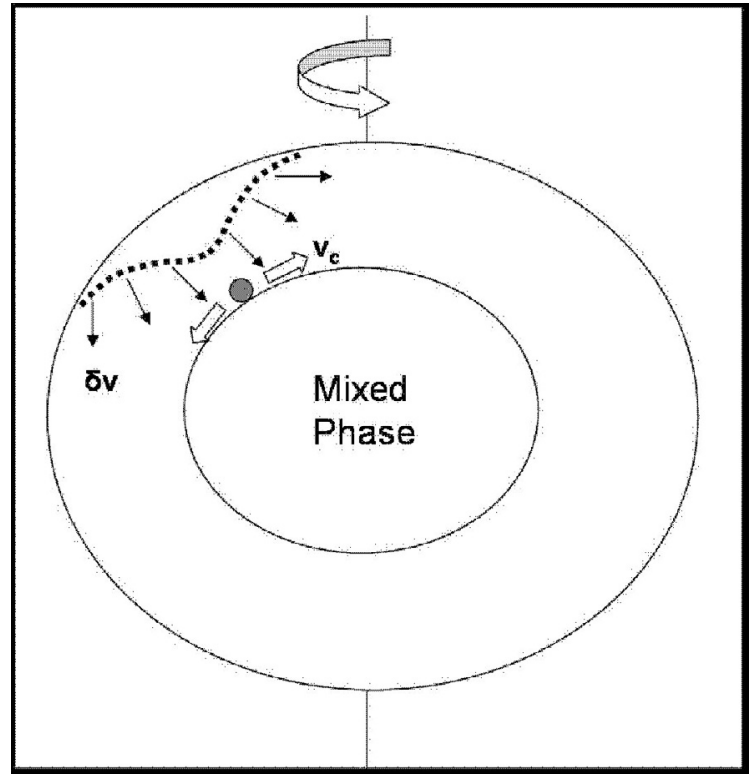

Fig. 7. The mini-collapse process. The nucleation site of the new drop of MP is indicated by the small gray circle, $\boldsymbol{v}_{c}$ indicates the velocity of the compression wave and $\delta v$ is the velocity of the infalling upper layers.

atomic-nuclear physics, while in our model the energy scale is related to hadronic physics.

In the model described here the emission of bursts of GWs is due to the collapse of the region involved in the formation of new MP. It is not easy to estimate the exact size of this region. Once a drop of quark matter develops in the zone where MP is being produced, the neighboring areas are probably triggered so that a relatively large fraction of the metastable layer will collapse. We can imagine that, after the mini-collapse, a compression wave develops and propagates from the site at which the new quark matter drop formed to the neighboring regions as shown in Fig. 7. The compression wave corresponds to a temporary overpressure that adds to the already existing pressure inside the metastable matter. To investigate if the propagation of the compression wave is able to trigger the formation of new structures of quark matter, we compare two timescales. The first timescale $t_{\text {trans }}$ corresponds to the duration of the overpressure $\delta p$ due to the compression wave on a specific point inside the metastable layer. It can be computed from the velocity of the compression wave $v_{\mathrm{c}}$ (which is the velocity of sound and it should be a fraction of the velocity of light) and the size of the compression wave itself $\Delta$, which should be of the same order as the radius of the newly formed quark matter drop whose formation originated the wave, i.e. $\Delta \sim 10 \mathrm{fm}$. In this way we get $t_{\text {trans }} \sim 10^{-20} \mathrm{~s}$, where only an estimate of the order of magnitude is needed. This timescale must be compared with the nucleation time $t_{\text {nucl }}$ that corresponds to the value of overpressure obtained adding the already existing overpressure in the metastable layer $\Delta p$ to the overpressure due to the compression wave $\delta p$. Assuming $\delta p \sim \Delta p$ and using the standard formalism we obtain $t_{\text {nucl }} \lesssim t_{\text {trans }}$. We conclude therefore that the conversion process can indeed propagate inside the metastable layer with a velocity marginally lower than $v_{\mathrm{c}}$. 
Within our model, it is not possible to give a precise estimate of the size of the region collapsing in a timescale of order $1 \mathrm{~s}$ or smaller. The extension of this region can vary from the whole metastable layer to a relatively small portion of the star. For instance, it is possible that the conversion will propagate only in the area in which new drops of QM are not too far from each other. The randomness of the size of the collapsing region is typical of quake phenomena. Once a fraction of the metastable layer has collapsed, the other parts will presumably follow the same fate in a timescale much shorter than the time needed to reach the critical value of the overpressure. Thus we expect a few in a relatively short period, while a much longer delay (of order years) separates the phases of quake activity. This feature is similar to the temporal distribution observed in soft-gamma repeaters (Cheng et al. 1995) and interpreted there as due to starquake activity (de Freitas Pacheco 1998). Interestingly, the temporal clustering of the events seems also to be a characteristic feature of the $\mathrm{GW}$ bursts emission (Coccia et al. 2004). A detailed analysis of the temporal structure of the GW bursters has been made in Dubath et al. (2005) where also the detection strategies of this type of signal is discussed.

\subsection{Energy of the GW bursts}

We will now try to estimate the energy of the GW bursts. It is not easy to provide a realistic approximation of this quantity since it would require a detailed analysis of the dynamics of the micro-collapse. In this section we will first present a rough estimate based on dimensional analysis and, after, a more quantitative discussion which will provide a lower limit on the energy released.

\subsubsection{Order of magnitude estimate}

The order of magnitude of the energy released can be estimated from the equation:

$E_{\mathrm{GW}}=M(\Delta R / R)^{2}$,

where $M$ is the mass in quadrupole motion and $\Delta R$ is the amplitude of the oscillation. In our model, the entire region of the star above the layer where the conversion occurs would participate in the quadrupolar motion, since the external regions have to readjust immediately after the new layer of MP is formed. Therefore, we can assume that the mass in quadrupolar oscillation is of the same order as the total mass of the star. Concerning $\Delta R$, it is of the order of the shrinking of the radius of the star due to formation of a new layer of MP. Using the toy model results, we can estimate that for each mini-collapse the variation of the radius of the star is of order 20-30 m, which corresponds to $\Delta R / R \sim 2 \div 3 \times 10^{-3}$. We can now put an upper limit on the energy released in GWs. Assuming that the oscillation induced by the phase transition is completely quadrupolar, the energy released would be of order $E_{\mathrm{GW}} \sim(0.5 \div 1) \times 10^{-5} M_{\odot}$. In the next Section we will study how the oscillation decomposes into the normal modes of the star and we will find that only a few percent of the total energy is associated with quadrupolar oscillations. On the other hand, a more realistic model should give larger variations of the radius for the same value of $\Delta \Omega / \Omega$. We can expect that in a more realistic calculation the upper limit on the energy released in GWs can be up to an order of magnitude larger, approaching $E_{\mathrm{GW}} \sim 10^{-4} M_{\odot}$.

\subsubsection{Decomposition of the perturbation into nonradial modes}

In this section we give a more quantitative estimate of the amplitude of the GW by explicitly performing the decomposition of the perturbation into the normal nonradial modes of the star. We first provide a "model" of the perturbation of the star's fluid induced by the conversion process. Then, after computing the spectrum of non-radial modes of the star, we decompose the perturbation on the basis of the normal oscillation modes, $\eta_{n l}$. In particular, we can compute the f-mode component $\eta_{\mathrm{f} 2}$ of the perturbation and the corresponding GW emission. This is important because the excitation of $\mathrm{f}$-mode is the most efficient way to produce GWs.

In order to model the perturbation of the pressure of the star during the micro-collapse, we use the toy model and assume that the perturbation corresponds to the overpressure already calculated in Sect. 3, since immediately after the conversion the overpressure reduces to zero. In Sect. 3 we computed the overpressure $\Delta P / P$ as a function of the effective radius $a$, for fixed values of $\Omega$ and $\Delta \Omega / \Omega$. Inverting the relation given by Eq. (6), we can write the overpressure as a function of $r$ and $\theta$. For small values of the deformation parameter $\epsilon$ the perturbation can be approximated by:

$\frac{\Delta P}{\rho}(a(r, \theta))=\left.\frac{\Delta P}{\rho}\right|_{\epsilon=0}+r \frac{\mathrm{d}(\Delta P / \rho)}{\mathrm{d} r} \epsilon P_{2}(\cos (\theta))$.

This corresponds to a decomposition of the perturbation into a radial term, which will not contribute to the emission of GWs, and a non-radial quadrupolar term, which will be further decomposed using the normal non-radial modes basis. In the following, while we compute the nonradial modes within a realistic model for the star, for the form of the perturbation $\Delta P / \rho$ we assume that it is given by the expression obtained within the toy model. This crude approximation can be overcome by computing the structure of a rotating star using a realistic EOS. A generic perturbation can be expanded as:

$$
\frac{\Delta P}{\rho}(r, \theta, \phi)=\sum_{n, l, m} c_{n l m} \eta_{n l}(r) Y_{l, m}(\theta, \phi),
$$

where $\eta_{n l}(r)$ are the eigenfunctions of the non-radial oscillation equation (see Unno et al. 1989). The index $n$ can indicate the fundamental $\mathrm{f}$ mode or the $\mathrm{p}$ and $\mathrm{g}$ modes. For simplicity, we have computed the spectrum of non-radial oscillations in the case of a non-rotating, Newtonian Hybrid Star, within the Cowling approximation and assuming that the Brunt-Vaisala frequency is vanishing. In this simplified case, g-modes have zero frequency, and the equation describing non-radial oscillations reduces to a Sturm-Liouville form.

In Fig. 8 we display the quadrupolar component of the perturbation, defined in Eq. (8), together with the $l=2$ eigenfunctions of the f-mode and of the first p-mode. It is now possible 


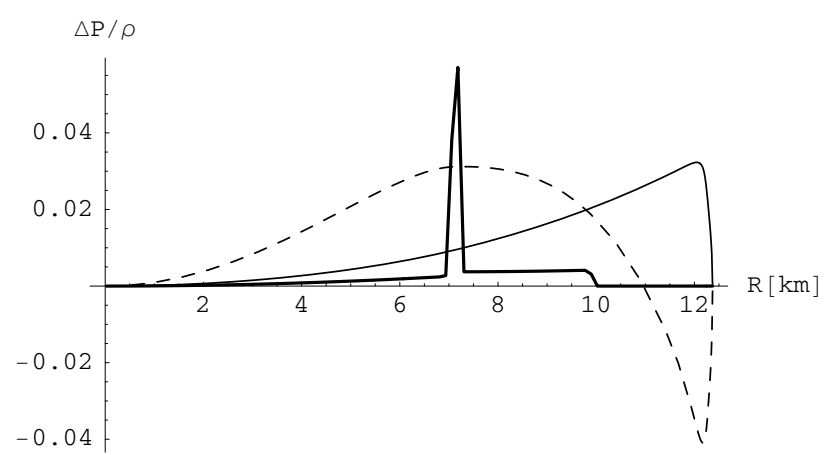

Fig. 8. The nonradial component of the perturbation $\Delta P / \rho$ (thick line) and the f-mode (continuous line) and first p-mode eigenfunctions (for $l=2$ ), as functions of the radius of the star.

to compute the f-mode component of the perturbation, which turns out to be $c_{\mathrm{f} 2} \simeq 0.05$.

The power emitted in GWs by the excitation of the f-mode can be estimated using the formula (Ipser \& Lindblom 1991):

$\left(\frac{\mathrm{d} E}{\mathrm{~d} t}\right)_{\mathrm{f} 2}=-\omega^{6} N_{2}\left|\Delta D_{20}\right|^{2}$

where the general expression for $N_{l}$ reads

$N_{l}=4 \pi \frac{G}{c^{2 l+1}} \frac{(l+1)(l+2)}{l(l-1)[(2 l+1) ! !]^{2}}$,

$\omega$ is the frequency of the mode and $\Delta D_{l m}$ is the mass multipole of the perturbed fluid. In our analysis we take into account only the quadrupolar contribution $(l=2)$. The mass multipoles read:

$\Delta D_{l m}=\int r^{l} \Delta \rho Y_{l m}^{*} \mathrm{~d}^{3} x$,

where $\Delta \rho$ is the perturbation of the mass density. The function $\Delta \rho$ can be expressed in terms of $\Delta P$, using the relation

$\Delta \rho=\frac{\rho}{\Gamma} \frac{\Delta P}{P}=\frac{\rho}{c_{\mathrm{s}}^{2}} \frac{\Delta P}{\rho}$

where $\Gamma$ is the adiabatic index and $c_{\mathrm{s}}$ is the sound velocity. Finally, for $\Delta P / \rho$ we use the $l=2$, f-mode component obtained from Eq. (9). Therefore the density multipole reads:

$(\Delta \rho)_{\mathrm{f} 2}=\frac{\rho}{c_{\mathrm{s}}^{2}} c_{\mathrm{f} 2} \eta_{\mathrm{f} 2} Y_{2 m}$.

Substituting this expression in Eqs. (10), (12) we get an estimate of the power emitted in GWs due to the excitation of the f-mode:

$\left(\frac{\mathrm{d} E}{\mathrm{~d} t}\right)_{\mathrm{f} 2} \sim 2.4 \times 10^{-7}\left(\frac{\omega / 2 \pi}{1 \mathrm{kHz}}\right)^{6} M_{\odot} / \mathrm{s}$.

In our estimate of the power of GWs emission we have considered up to now only the contribution of the mass quadrupole, but it is necessary also to take into account the contribution associated with the current multipole, given by the expression:

$\delta J_{l m}=\frac{2}{c} \sqrt{\frac{l}{l+1}} \int r^{l}(\delta \rho \boldsymbol{v}+\rho \delta \boldsymbol{v}) \cdot \boldsymbol{Y}_{l m}^{B *} \mathrm{~d}^{3} x$, where $\boldsymbol{Y}_{l m}^{B *}$ are the magnetic spherical harmonics. In Eq. (16) two terms are present: one, $\boldsymbol{v} \delta \rho$, is associated with the velocity of the fraction of the fluid which undergoes the density fluctuation. This term is suppressed relative to the one already estimated, due to the presence of a factor $v / c$ which is obviously always smaller than one. The second term, $\rho \delta \boldsymbol{v}(\boldsymbol{r})$, corresponds to the variation of the velocity of all fluid elements inside the star. In our model, $\delta \boldsymbol{v}(\boldsymbol{r})$ is associated with the rearrangement of the external parts of the star, immediately after the formation of a new layer of MP. It is very difficult to estimate $\delta \boldsymbol{v}(\boldsymbol{r})$, and in particular its dependence on the position inside the star. We can assume that all regions above the conversion layer will move, as in Fig. 7. This term of the current multipole can be a more efficient source of GWs than the mass quadrupole, which is relatively small because $\Delta P / \rho$ is non vanishing only in a narrow region. On the other hand, the integral that defines the current multipole extends over a larger volume. From simple order of magnitude estimate we found that its contribution to the emitted power can be up to one order of magnitude greater than the one associated with the mass multipole.

\subsubsection{Frequency, time duration and amplitude of the GW bursts}

For the value of the frequency of the emitted GW burst, in the simplified Newtonian scheme we have adopted, the eigenfrequencies of the f-mode and the p-mode are respectively of 3 and $8 \mathrm{kHz}$. Calculations of these frequencies in full General Relativity give smaller values, typically of the order of $2 \mathrm{kHz}$ for the f-mode (Benhar et al. 2004). The corresponding duration is $\tau_{\mathrm{f}} \sim 0.18 \mathrm{~s}$ (Benhar et al. 2004) and therefore the total energy emitted by the f-mode is of the order of $E_{\mathrm{f}} \sim$ $1.2 \times 10^{-6} M_{\odot}$.

In the estimate of the f-mode frequency we have up to now neglected the effect of rotation. It is known that in a rotating star the eigenfrequencies are shifted towards lower values (Ferrari et al. 2004). In particular, the frequency of the f-mode reduces to a value of the order of $1 \mathrm{kHz}$ for fast rotating stars.

From the emitted power it is possible to estimate the initial amplitude $h_{0}$ of the GW using the equation (de Freitas Pacheco 1998):

$h_{0}=\left(-2 \frac{\mathrm{d} E}{\mathrm{~d} t}\right)^{1 / 2} \frac{1}{D \omega_{\mathrm{f}}}$,

where $D$ is the distance of the source. We therefore obtain

$h_{0} \sim 3 \times 10^{-21}\left(\frac{\omega / 2 \pi}{1 \mathrm{kHz}}\right)^{2}\left(\frac{1 \mathrm{kpc}}{D}\right)$.

The time dependence of the GW is given by

$h(t)=h_{0} \exp ^{-\left(t / \tau_{\mathrm{f}}-i \omega_{\mathrm{f}} t\right)}$

and the Fourier transform is therefore:

$\tilde{h}(\omega)=\frac{h_{0} / \tau_{\mathrm{f}}}{1 / \tau_{\mathrm{f}}^{2}+\left(\omega-\omega_{\mathrm{f}}\right)^{2}}$.

If $\omega-\omega_{\mathrm{f}} \sim 1000 \mathrm{~s}^{-1}$ then $\tilde{h} \sim\left(10^{-6} \mathrm{~s}^{2}\right) h_{0} / \tau_{\mathrm{f}}$. Instead if $\omega-\omega_{\mathrm{f}} \sim$ $1 / \tau_{\mathrm{f}}$ then $\tilde{h} \sim h_{0} \tau_{\mathrm{f}} / 2$. 
We can compare our results with the characteristics of the signal detected by NAUTILUS and EXPLORER. The experimental value of the Fourier transform of the amplitude is $\tilde{h} \sim 2 \times 10^{-21}$ s. Clearly our model can approach this value only if the frequency $\omega_{\mathrm{f}}$ is very near to the resonance frequency of the two gravitational bars, which is of the order of $1 \mathrm{kHz}$. Even in this case, to obtain in our model an amplitude comparable to the experimental one it is necessary to assume that the emitted power is much larger, which maybe due to the current multipole term and/or to the effect of using a more realistic model.

Finally, we want to stress that, even using the toy model, the energy released in GW bursts is greater than the energy of the bursts in other models of GW bursters, e.g. soft gamma repeaters. There, energy of $\sim 10^{-10}-10^{-9} M_{\odot}$ can be released, but only under the assumption that all the elastic stress is completely converted into GW bursts (de Freitas Pacheco 1998).

\section{Conclusions}

We now discuss the phenomenological relevance of the proposed model. For the stellar objects that can be possible candidates for our model, if we assume that all neutron stars are born with high angular velocity, they will all enter the instability window as described in the first scenario. Taking a neutron star production rate of the order of 0.02 per year in our galaxy, and assuming the possibility of detecting GW bursts up to a distance of about $1 \mathrm{kpc}$, the probability of finding an active burster in this region is a few percent, if the total duration of the emission phase is near 50 years. If the "recycling" described in the second scenario is taken into account, the probability can be larger. A precise estimate of the probability would require a precise knowledge of the number of accreting millisecond pulsars, what is not known at the moment.

An important feature of the model discussed here is that no neutrino signal is expected in detectors as LVD (in the Gran Sasso National Laboratory) or even in more massive detectors like Super Kamiokande. In our model an energy $E_{\text {trans }} \sim$ $10^{-4} M_{\odot}$ is deposited inside the star, near the metastable layer, during the transition. The neutrinos produced by URCA processes have typical energies of a few $\mathrm{MeV}$ and they will scatter many times inside the star, degrading their energy before escaping. Therefore the emitted neutrinos have energies below the threshold of neutrino detectors.

Finally, let us discuss possible signatures of the mechanism proposed here. Concerning the number of epochs during which bursts are emitted, we can expect that it will decrease from a few active periods in ten years to a few periods in the next hundred years, because the angular velocity decreases by roughly the same amount in the first ten years as in the following hundred years. The amplitude of the signal should also decrease because, as shown in Figs. 4 and 5, when the angular velocity is lower, the variation of the radius of the star is also smaller. Both these features are related to the draining of the burst energy source which, in this model, is ultimately the rotational energy of the star.

Acknowledgements. It is a pleasure to thank E. Coccia, G. Fiorentini and M. Maggiore for very useful discussions. A special thank to
A. Ortolan for suggesting the possible connection between sudden variations of the star structure and the emission of short GW bursts.

\section{References}

Abbott, B., et al. 2004, Phys. Rev. D, 69, 082004

Andersson, N. 1998, ApJ, 502, 708

Andersson, N., Jones, D. I., Kokkotas, K. D., \& Stergioulas, N. 2000, ApJ, 534, L75

Andersson, N., Jones, D. I., \& Kokkotas, K. D. 2002, MNRAS, 337, 1224

Astone, P., Babusci, D., Bassan, M., et al. 2002, Class. Quant. Grav., 19,5449

Astone, P., D’Agostini, G., \& D’Antonio, S. 2003a, Class. Quant. Grav., 20, S769

Astone, P., Babusci, D., Bassan, M., et al. 2003b, Class. Quant. Grav., 20, S785

Benhar, O., Ferrari, V., \& Gualtieri, L. 2004, Phys. Rev. D, 70, 124015

Berezhiani, Z., Bombaci, I., Drago, A., Frontera, F., \& Lavagno, A. 2003, ApJ, 586, 1250

Berger, M. S., \& Jaffe, R. L. 1987, Phys. Rev. C, 35, 213

Bildsten, L., \& Ushomirsky, G. 1999, ApJ, 529, L33

Bombaci, I., Parenti, I., \& Vidana, I. 2004, ApJ, 614, 314

Cheng, B., Epstein, R., Guyer, R., \& Young, A. 1995, Nature, 382, 518

Chubarian, E., Grigorian, H., Poghosyan, G. S., \& Blaschke, D. 2000, A\&A, 357, 968

Coccia, E., Dubath, F., \& Maggiore, M. 2004, Phys. Rev. D, 70, 084010

de Freitas Pacheco, J. A. 1998, A\&A, 336, 397

Drago, A., Lavagno, A., \& Pagliara, G. 2004, Phys. Rev. D, 69, 057505

Drago, A., Lavagno, A., \& Pagliara, G. 2005, Phys. Rev. D, 71, 103004

Dubath, F., Foffa, S., Gasparini, M. A., Maggiore, M., \& Sturani, R. 2005, Phys. Rev. D, 71, 124003

Ferrari, V., Gualtieri, L., Pons, J. A., \& Stavridis, A. 2004, MNRAS, 350,763

Finn, L. S. 2003, Class. Quant. Grav., 20, L37

Friedman, J. L., \& Morsink, S. M. 1998, ApJ, 502, 714

Glendenning, N. 1997, Compact Stars (Springer-Verlag)

Glendenning, N. K., Pei, S., \& Weber, F. 1997, Phys. Rev. Lett., 79, 1603

Harko, T., Cheng, K. S., \& Tang, P. S. 2004, ApJ, 608, 945

Heiselberg, H., \& Hjorth-Jensen, M. 1998, Phys. Rev. Lett., 80, 5485

Heyl, J. S. 2002, ApJ, 574, L57

Iida, K., \& Sato, K. 1998, Phys. Rev. C, 58, 2538

Ipser, J. R., \& Lindblom, L. 1991, ApJ, 373, 213

Kinney, J. B., \& Mendell, G. 2003, Phys. Rev., D67, 024032

Levin, Y. 1999, ApJ, 517, L328

Levin, Y., \& Ushomirsky, G. 2001, MNRAS, 322, 515

Lindblom, L., \& Owen, B. J. 2002, Phys. Rev. D, 65, 063006

Madsen, J. 2000, Phys. Rev. Lett., 85, 10

Miniutti, G., Pons, J. A., Berti, E., Gualtieri, L., \& Ferrari, V. 2003, MNRAS, 338, 389

Owen, B. J., Lindblom, L., Cutler, C., et al. 1998, Phys. Rev. D, 58, 084020

Reisenegger, A., \& Bonacic, A. A. 2003, Phys. Rev. Lett., 91, 201103

Unno, W., Osaki, Y., Ando, H., Saio, H., \& Shibahashi, H. 1989, Nonradial Oscillation of Stars (University of Tokyo press)

Wagoner, R. V. 2002, ApJ, 578, L63 